\title{
Geometrical modelling of pulsed Laser ablation of high performance metallic alloys.
}

\author{
D. Cha ${ }^{a}$, D. Axinte ${ }^{a *}$, J. Billingham ${ }^{b}$ \\ ${ }^{a}$ Machining and Condition Monitoring Group, Faculty of Engineering, University of Nottingham, NG7 \\ 2RD, UK \\ ${ }^{\mathrm{b}}$ School of Mathematical Sciences, University of Nottingham, NG7 2RD, UK
}

\begin{abstract}
Modelling of Pulsed Laser Ablation (PLA) for the prediction of complex geometries has generally achieved limited success when aimed at large structures resulting from a high number of overlapped pulses, in particular for the ablation of metallic materials, where a significant volume of molten and re-deposited material can be present. In order to extend the capabilities of process simulation for surface prediction of PLA, this paper presents a novel problem formulation that takes into consideration the behaviour of the ejected/redeposited melt as well as the non-linear interaction between successive pulses when a laser beam is scanned along a given path. This results in a simplified mathematical framework capable of predicting features with good accuracy and low computational cost. The evolution of the depth/height at any point on the surface can be described by the convolution of a radially-varying function that represents the steady state ablation footprint (which includes also material redeposition) created by a pulsed laser scanned across the workpiece scaled according to pulse separation distance (i.e. feed speed). The model also reveals some interesting dynamics of the behaviour of redeposited material, which appears to have a lower removal threshold compared to the virgin material. This can be taken into account in a modified model formulation by introducing a linear scaling coefficient for the ablation function. Validation of the model on $\mathrm{Ni}$ - and Tisuperalloy for both the prediction of single trenches (i.e. scanning along straight path) at constant and variable feed speed, and overlapped trenches, is performed with an average error of less than $10 \%$. The framework presented in the paper could provide a valuable step forward in process modelling of PLA for real-world industrial applications.
\end{abstract}

Keywords: pulse laser ablation; geometrical modelling; redeposition, high performance alloy 


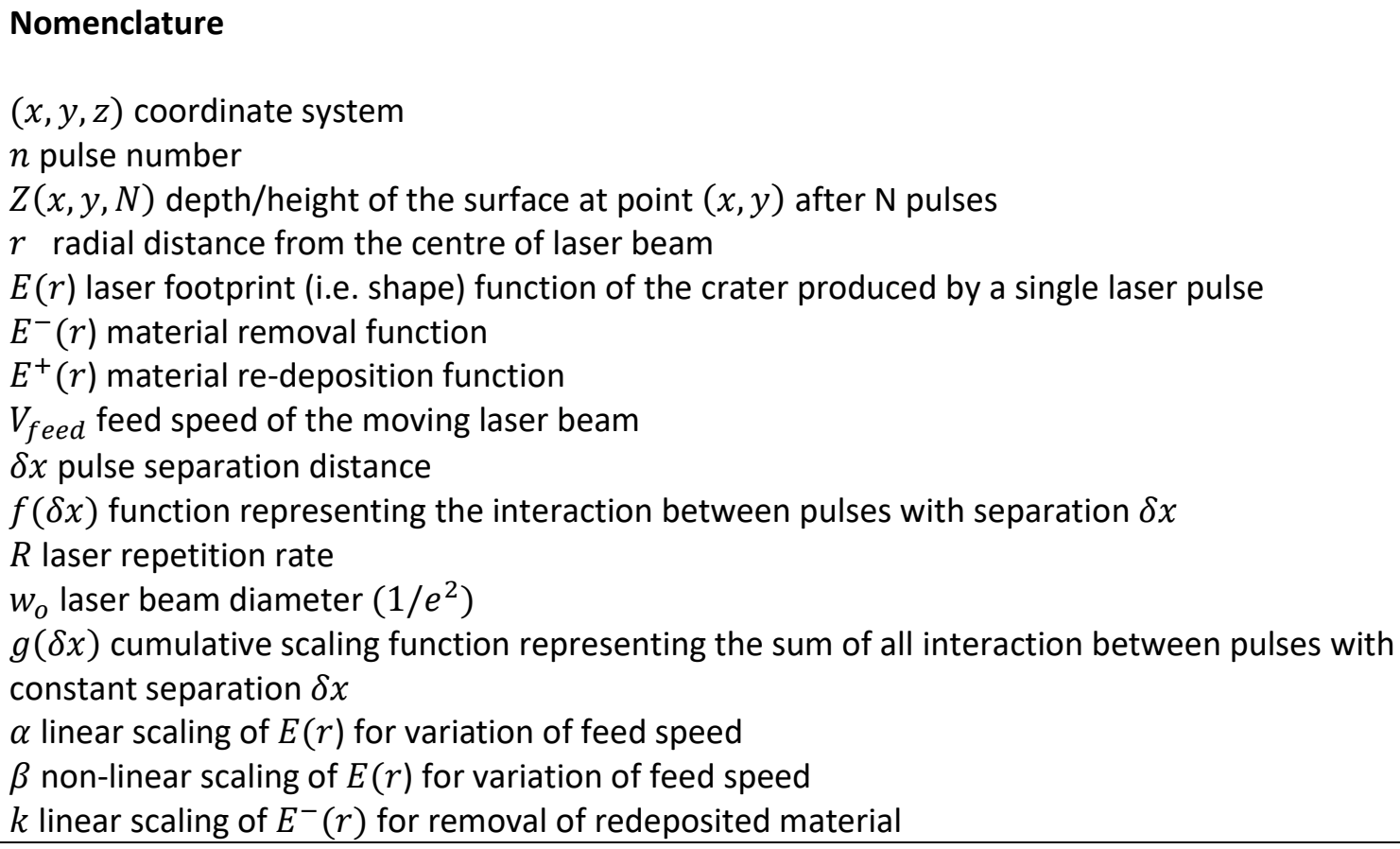




\section{Introduction}

In recent years, the use of nanosecond pulsed lasers for micro-machining applications has gained widespread use in industry. In comparison to traditional manufacturing techniques, there are significant advantages, such as the ability to machine complex structures within micron-scale tolerances [1], and to cut virtually any material [2]. In addition, compared to other types of laser (ultrafast, CW), nanosecond laser ablation currently offers a good compromise between low surface damage (i.e. Heat Affect Zone, HAZ) and high volume throughput [3].

In general, Pulsed Laser Ablation (PLA) removes material using a highly focused laser beam, with intensity high enough $\left(10^{\wedge} 8-10^{\wedge} 11 \mathrm{~W} / \mathrm{cm}^{2}\right)$ to vaporise material nearly instantly [4]. At industrial levels, highly automated systems are often used, where the laser beam is scanned along a 2D path via a galvo scanner, allowing control of the desired depth of a machined feature by changes in scanning speed of the laser beam (i.e. feed speed). This is advantageous compared to control of the process by changes in power, as it allows a much more flexible and robust way of producing complex 2- and 3-D features as it is easy to continuously control the ablated material via beam path planning (including feed speed adjustment) using CNC of the machine system.

Being a time dependent process (amount of removed material depends on time exposure of the beam over a particular area), PLA often requires experimental trial-and-error calibration to generate a desired workpiece geometry. Complete physical modelling of the process represents a challenging and computationally expensive task, often infeasible for large structures. The physical phenomena that occur during PLA span several time scales, ranging from picosecond (photon absorption [5], thermalization [5]), to nanosecond (phase changes [6], plasma formation [6]) microsecond (ejection [7], re-solidification [6]), and all states of matters (solid, liquid, vapour and plasma). Often, physical models/simulations must rely on sets of assumption, such as constant material properties [8], a 1 or 2 dimensional domain [9] [10], or simplified plasma dynamics [11], to avoid excessive computational time.

Additional issues are related to the presence of non-linear interactions between successive laser pulses, which renders most of the current approaches to predict real output geometries inconclusive in industrial applications with thousands of overlapped pulses. This also presents a problem for most mathematical models [12][13][14][15], as they are often based on calibration from the resultant crater (i.e. footprint) produced by a single laser pulse.

Experimentally, a reduction in ablation threshold in multi-pulse irradiation of a target surface has been observed for a variety of different materials. In metals, this effect has been linked to the accumulation of thermally-induced plastic strain [16], from which a cumulative equation of the form $F_{t h}(N)=$ $F_{t h}(1) N^{S-1}$ has been formulated to link the resultant fluence threshold $F_{t h}(N)$ after the $\mathrm{N}^{\text {th }}$ pulse with the single pulse fluence threshold $F_{t h}(1)$, with incubation coefficients $S$ ranging from 0.6-0.9 [17][18]. More recent investigations have revealed that the origin of this incubation could be the formation of micro/nano pores below the surface due to the rapid quenching of molten material, which drastically reduces the mechanical integrity of the redeposited material, and therefore translates to an increase in material removal due to spallation [19]. 


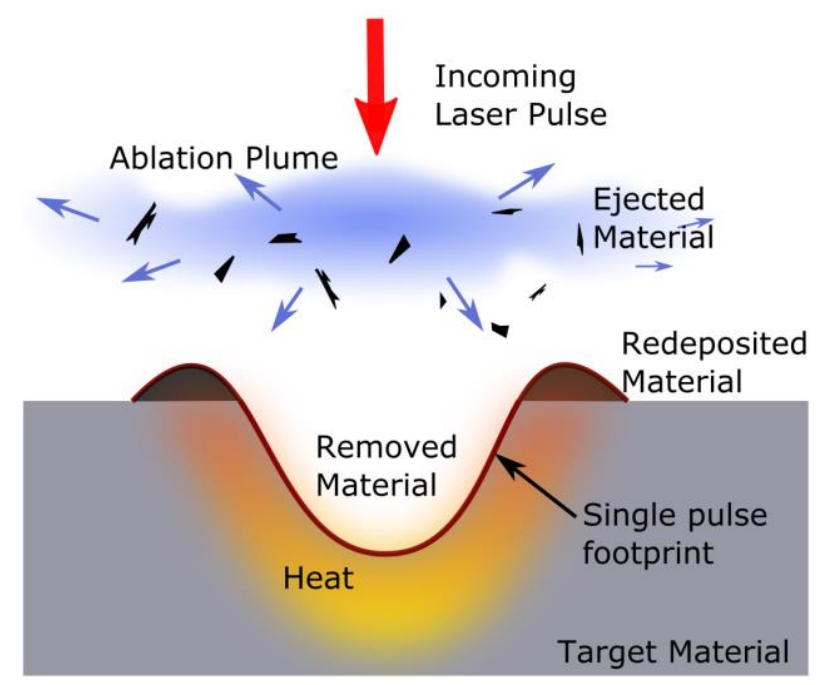

Fig. 1. Schematic of the Pulsed Laser Ablation (PLA) process and the resulting laser footprint on the target material (including removed and redeposited material)

An additional source of non-linearity during interaction between laser pulses originates from the ejected material that constitutes the ablation plume (see Fig.1) [20]. The formation of plasma, which partially absorbs the incoming energy from the laser pulse, is a common occurrence in the nanosecond regime [20]. Furthermore, delayed ejection of particles, up to $1 \mu \mathrm{s}$ after the end of a pulse has been shown to be present [7]. This is a significant issue, especially for the high repetition rate lasers (i.e. short intra-pulse time separation) [21] that industry is currently progressing towards. Two competing mechanisms, the increase of absorbance due to intra-pulse heat conservation and the reduction of incoming energy due to plume shielding, are present and dominate at different values of repetition rates [21].

This is further complicated when considering a moving laser source. In this case, the effect of the separation distance between pulses (i.e. feed speed) can assume a major role in dictating the degree of interaction. Some attempts at predicting the non-linear effects when a pulsed laser is scanned along a straight path have been made [22][23] mostly focusing on the effect of material incubation, but have been less successful to account for all sources of non-linearity previously discussed and this would result in limited ability to predict accurately the workpiece surface topography.

Recently, a novel framework has been proposed to allow simulation of any generic energy beam process (PLA, FIB, water-jet) from a simple set of calibration trials, while maintaining good accuracy and low computational cost [24]. The application of this model to PLA [25] has successfully demonstrated the ability of this approach to predict and account for the non-linear effects present during ablation only of particular classes of material (graphites and diamonds) that do not re-deposit. However, when considering laser micro-machining of metals, the model is not able to account for the presence of molten material, often present in significant quantity during nanosecond ablation due to the relatively long pulse duration. The dynamics of the molten material is influenced by the recoil pressure originating from the impingement of the expanded plume on the temporary molten pool created at the bottom of a pulse crater, with an additional contribution from surface tension [26]. Some of the molten material is ejected to contribute to the formation of the ablation plume [20], while the rest sticks to the surface giving rise to the characteristic redeposition pile-ups features produced on the side of an ablation crater (see Fig. 1). 
Although some attempts at modelling material re-deposition have been presented [27][28][29], their predictions fail to account for the non-linearity intrinsically present in the ablation process, and have generally had limited success when used for geometrical prediction of complex features.

In order to extend current capabilities of process simulation for pulsed laser ablation, this study aims to present a computationally-cheap model able to predict surface geometry after PLA of metallic materials which experience redeposition, whilst simultaneously accounting for the non-linear interaction between successive pulses. In particular, after a simple calibration procedure based on a limited set of experimental trials, the model is able to predict structures produced by a scanned laser beam, such as single trenches (i.e. scanning along a straight path) and overlapped trenches. The novelty of the model lies in its ability to capture the non-linear effects and the evolution of redeposited material pile up for a number of different scenarios. This is a significant advancement over current models, and could provide a valuable step forward in process modelling to enable the use of PLA as a method for machining true $2.5 / 3 \mathrm{D}$ free-forms in metallic materials that present redeposition.

\section{Mathematical model}

This section develops the mathematical framework for modelling of PLA of metallic materials which redeposit. The model is presented as two parts: section 3.1 lays the groundwork for the modelling of simple features produced by a laser beam scanned along a straight path (i.e. single trenches), while section 3.2 proposes a model for the prediction of overlapped trenches.

\subsection{Single Trenches Model}

We consider the simple case of a laser pulse from a laser source with given wavelength, power, repetition rate $R$ and beam diameter $w_{o}$, incident on a target surface $z=Z(x, y, n)$ of a given material, where $n$ is the pulse number, and with initial condition $Z(x, y, 0)=0$. The 2D surface describing the variation of the depth (for removal)/height (for redepositon) after the pulse can be expressed as

$$
Z(x, y, 1)=E\left(\sqrt{x^{2}+y^{2}}\right)=E(r)=E^{+}(r)+E^{-}(r)
$$

where $E(r)$ is a radial function that represents the footprint (i.e. shape) of the crater produced by a single laser pulse (see Fig. 1), which includes material removal $E^{-}(r) \leq 0$ via vaporization and melt displacement/ejection, representing the dominant processes in nanosecond PLA of metals [30], and material re-deposition, $E^{+}(r)>0$ (see Fig. 2).

We now consider the case of a second identical pulse incident on the surface at later time, displaced, for example, in the $x$ direction by $\delta x=\frac{V_{\text {feed }}}{R}$, where $V_{\text {feed }}$ is the feed speed (i.e. scanning speed) of the moving laser source. The change in surface depth/height due to the $2^{\text {nd }}$ pulse can be expressed as

$$
\begin{aligned}
\delta Z(x, y, n, \delta x)= & \delta Z(x, y, 2, \delta x) \\
= & E^{+}\left(\sqrt{(x-\delta x)^{2}+y^{2}}\right) f^{+}(\delta x)+E^{-}\left(\sqrt{(x-\delta x)^{2}+y^{2}}\right) f^{-}(\delta x),
\end{aligned}
$$

where $f^{-}(\delta x)$ and $f^{+}(\delta x)$ are scaling functions that relate to removal and redeposition, and model the effect of the interactions between the $2^{\text {nd }}$ and $1^{\text {st }}$ pulses as a function of separation distance $\delta x$, assuming that $f^{+}(\delta x)$ and $f^{-}(\delta x)$ only scale the footprint function $E(r)$ in the $z$ direction. 
The resultant surface after the two overlapped pulses will therefore be

$$
Z(x, y, 2)=Z(x, y, 1)+\delta Z(x, y, 2, \delta x)
$$

For the $n^{\text {th }}$ pulse of a scanned laser moving with constant $V_{\text {feed }}$ (i.e. constant $\delta x$ ) along a straight path, we need to consider the interaction with all preceding pulses. In doing this, we assume that the interaction between pulses can extend further than the diameter of a single pulse (for $\delta x>w_{o}$ ), to account for mechanisms such as plume shielding and heat accumulation which can still affect pulses close to each other but not overlapping. This can be expressed as

$$
\begin{aligned}
& \delta Z^{+}(x, y, n, \delta x)=E^{+}\left(\sqrt{(x-(n-1) \delta x)^{2}+y^{2}}\right)\left[f^{+}(\delta x) f^{+}(2 \delta x) f^{+}((n-1) \delta x)\right], \\
& \delta Z^{-}(x, y, n, \delta x)=E^{-}\left(\sqrt{(x-(n-1) \delta x)^{2}+y^{2}}\right)\left[f^{-}(\delta x) f^{-}(2 \delta x) f^{-}((n-1) \delta x)\right] .
\end{aligned}
$$

Effectively, we can assume that the footprint of the $n^{\text {th }}$ pulse will be influenced just by a limited subset of the preceding pulses when considering a large number of overlapped pulses. That is

$$
\lim _{n \rightarrow \infty} f^{+}(n \delta x)=1, \quad \lim _{n \rightarrow \infty} f^{-}(n \delta x)=1 .
$$

Eq. 5

In the case of constant $V_{\text {feed }}$ (i.e. constant $\delta x$ ), we define a cumulative scaling function $g(\delta x)$ representing the sum of all interaction from pulses with constant separation $\delta x$ as

$$
g^{+}(\delta x)=\prod_{n=1}^{\infty} f^{+}(n \delta x), \quad g^{-}(\delta x)=\prod_{n=1}^{\infty} f^{-}(n \delta x) .
$$

We can therefore rewrite Eq. 3 as

$$
\begin{aligned}
\delta Z(x, y, n, \delta x)= & E^{+}\left(\sqrt{(x-(n-1) \delta x)^{2}+y^{2}}\right) g^{+}(\delta x) \\
& +E^{-}\left(\sqrt{(x-(n-1) \delta x)^{2}+y^{2}}\right) g^{-}(\delta x)
\end{aligned}
$$

Experimentally, it has been found that for straight trenches (i.e. scanning along straight path) obtained at constant feed speed (i.e. constant separation $\delta x$ ), Eq. 7 is sufficient to describe the evolution of the surface, and a power law relationship has been found to describe reasonably well a wide range of separation distances (i.e. feed speed), with

$$
g^{+}(\delta x)=\left[\frac{\alpha^{+}}{\delta x^{\beta^{+}}}\right], \quad g^{-}(\delta x)=\left[\frac{\alpha^{-}}{\delta x^{\beta^{-}}}\right]
$$

Here $\beta^{+}$and $\beta^{-}$characterise the coefficient of the non-linear variation of material removal $\left(E^{-}\right)$and material redeposition $\left(E^{+}\right)$as a function of $\delta x$, and $\alpha^{+}$and $\alpha^{-}$the relative scaling factor. These parameters need to be calibrated for each material-laser (wavelength, power, repetition rate and beam diameter) combination within the feed speed range considered (remembering that $\delta x=$ $\left.V_{\text {feed }} / R\right)$. 
Finally, the workpiece surface $Z(x, y, N)$ obtained after $N$ pulses is

$$
Z(x, y, N)=Z(x, y, 1)+\sum_{n=2}^{N} \delta Z(x, y, n, \delta x)
$$

with model parameters $E(r), \alpha^{+}, \alpha^{-}, \beta^{+}$and,$\beta^{-}$that need to be calibrated experimentally (discussed later in section 4.2).

If we ignore the initial transient response (see Fig. 2), the interaction between successive pulses will reach a steady state once enough pulses have been deposited, and, by calibrating the model parameters in this region, Eq. 9 can then be used to predict single trenches produced by a scanned laser beam at constant feed speed and at variable feed speed, provided that the variation of speed is slow.

As will be shown later, this approximation is reasonable for modern high repetition rate lasers where each feature produced is the result of hundreds or more overlapped pulses, and variation of feed speeds is slow.

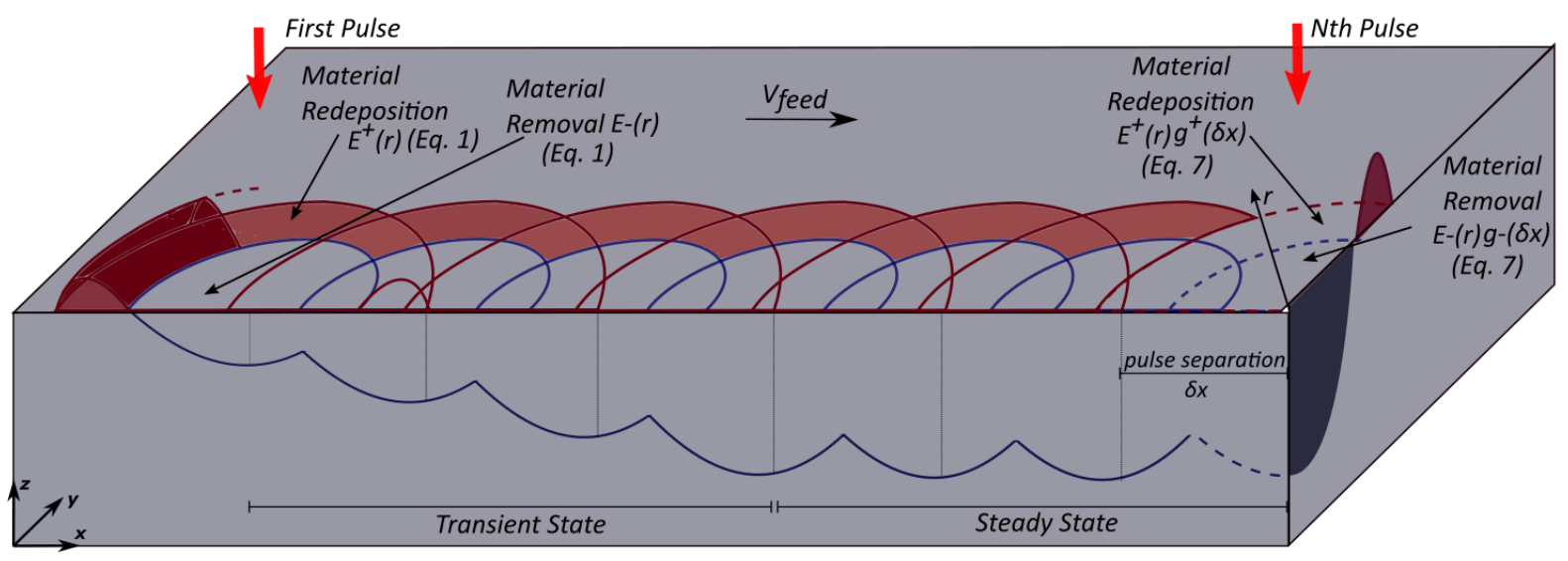

Fig. 2. Diagram of geometrical modelling of PLA for single trenches produced by a scanned laser beam that includes the material removal and redeposition zone, highlighting the model assumption of a steady state process after a large number of pulses have been deposited on the surface.

\subsection{Overlapped Trenches Model}

When considering overlapped trenches (Fig. 3), it has been found that the current model underpredicts the amount of material removed, in particular, material that has been redeposited by previous pulses; this is likely caused by the effect of the laser on the surface causing changes in material properties. For example, during ablation of $\mathrm{Ti}$ - and $\mathrm{Ni}$ - superalloy, it is known that the properties of redeposited material are different from those of the bulk due to changes in microstructure and chemical composition (i.e. oxidation) [31][32], as well as the imperfect adhesion with the surface [19][31], which could influence the ablation process and result in an increase of material removal, either due to reduced mechanical integrity [19] or increase of absorptance [32][33].

These phenomena could represent a significant source of error, since the volume of redeposited material is not negligible, in particular in those regions where redeposition pile-ups occurs (see Fig. 3). 


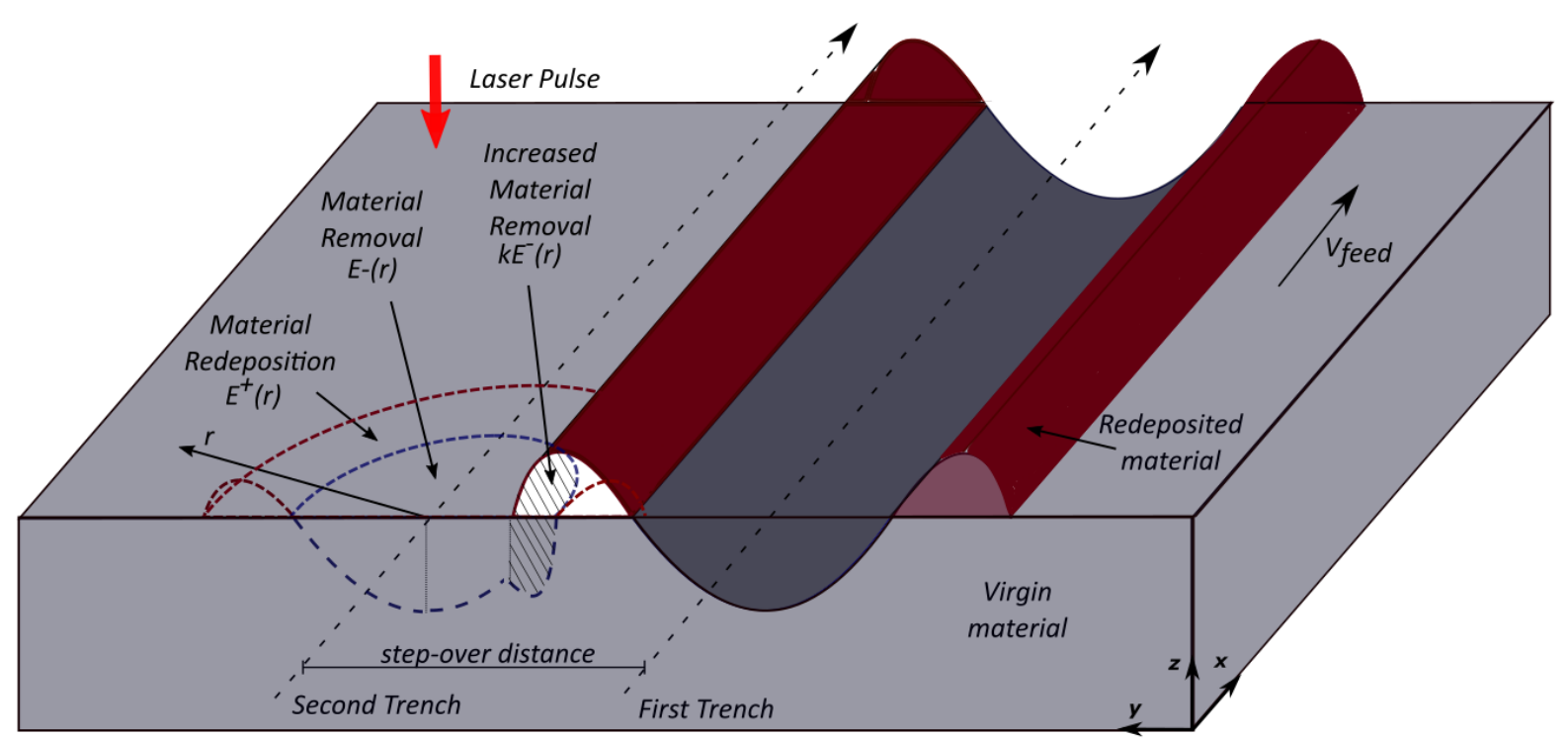

Fig. 3. Diagram of geometrical modelling of PLA for overlapped trenches produced by a scanned laser beam that includes the material removal and redeposition zone, highlighting the increase of material removal when ablating material redeposited by previous pulses.

The current model is therefore modified to add a linear increase of the material removal when ablating the portion of the footprint where redeposited material is already present. This modification requires tracking of material movement and identity (i.e. virgin or redeposited) and can be formulated as follows:

- Consider two surfaces $z=Z_{1}(x, y, n)$ and $z=Z_{2}(x, y, n)$, with initial conditions $Z_{1,2}(x, y, 0)=0$, where $Z_{1}$ represents the height of the virgin material and $Z_{2}$ that of redeposited material.

- Consider the $n^{t h}$ pulse of the laser with footprint $E(r)$, with $E^{-}(r)$ representing the material removal and $E^{+}(r)$ the material redeposition (such that $E(r)=E^{-}(r)+E^{+}(r)$ ).

- For the $n^{\text {th }}$ pulse, if $Z_{2}(x, y, n)>0$, that is, redeposited material is being removed from $Z_{1}(x, y, n)$ (see Fig. 3 ), the material removal footprint function $E^{-}(r)$ is scaled linearly by a coefficient $k=k_{1}$ (this assumption will be shown later as valid during the experimental validation), with

$$
\delta Z_{2}^{-}(x, y, n, \delta x)=k_{1} E^{-}\left(\sqrt{(x-(n-1) \delta x)^{2}+y^{2}}\right) g^{-}(\delta x)
$$

- If $Z_{2}(x, y, n)=0$, that is, virgin material is being removed from $Z_{1}(x, y, n)=0$ the material removal footprint function $E^{-}(r)$ behaves normally, with $k=1$, as

$$
\delta Z_{1}(x, y, n, \delta x)=E^{-}\left(\sqrt{(x-(n-1) \delta x)^{2}+y^{2}}\right) g^{-}(\delta x)
$$

- Subsequently, material is redeposited with

$$
\delta Z_{2}^{+}(x, y, n, \delta x)=E^{+}\left(\sqrt{(x-(n-1) \delta x)^{2}+y^{2}}\right) g^{+}(\delta x)
$$


- The overall change of surface $Z_{2}$ for the $n^{\text {th }}$ pulse is therefore

$$
\delta Z_{2}(x, y, n, \delta x)=\delta Z_{2}^{-}(x, y, n, \delta x)+\delta Z_{2}^{+}(x, y, n, \delta x)
$$

- The two surfaces $Z_{2}$ and $Z_{1}$ are updated before the next pulse is calculated, with

$$
\begin{aligned}
& Z_{1}(x, y, n)=Z_{1}(x, y, n-1)+\delta Z_{1}(x, y, n, \delta x), \\
& \quad Z_{2}(x, y, n)=Z_{2}(x, y, n-1)+\delta Z_{2}(x, y, n, \delta x) .
\end{aligned}
$$

- The final surface $Z(x, y, N)$, obtained after $N$ pulses, is the sum of the two surfaces calculated, so that

$$
Z(x, y, N)=Z_{1}(x, y, N)+Z_{2}(x, y, N)
$$

with model parameters $E(r), \alpha^{+}, \alpha^{-}, \beta^{+}, \beta^{-}$and $k_{1}$ that need to be calibrated experimentally within a given feed speed range for each material-laser (wavelength, power, repetition rate and beam diameter) combination.

Finally, Eq. 16 can be used for prediction of ablated and redeposited surfaces for single and overlapped trenches produced by a scanned laser beam, while also considering the increased material removal of material previously redeposited. The calibration of the model parameters is discussed later in section 4.2 .

\section{Experimental Methodology and Model Calibration}

\section{1 Experimental Methodology}

A nanosecond fibre laser (SPI-G3-HM) with a wavelength of $1064 \mathrm{~nm}$, a pulse duration of 50ns (FWHM) and pulse repetition frequency of $35 \mathrm{kHz}$ is used in this study. The laser beam is focused using a 100 $\mathrm{mm} \mathrm{f}$-theta lens to produce a Gaussian beam of $52.5 \mu \mathrm{m}$ diameter $\left(w_{o}, 1 / e^{2}\right.$ definition) as measured with a beam profiler (CMOS-1203, Cinogy Technologies), with a beam quality factor $M^{\wedge} 2$ of $<1.6$. The average power of the laser can be controlled in the range $0-17.2 \mathrm{~W}$, which corresponds to a fluence of up to $22.7 \mathrm{~J} / \mathrm{cm}^{2}$ at the focal plane.

Movement of the laser beam-target workpiece is achieved using an Aerotech 5-axis system comprised of a 3-axis motorized stage for positioning the workpiece (1um accuracy), and a 2 -axis galvo scanner (AGV10HP) for movement of the beam on the focal plane. The maximum error in the velocity is estimated from the system control feedback as $4 \mathrm{~mm} / \mathrm{s}$. A schematic of the above described experimental setup is shown in Fig. 4. 


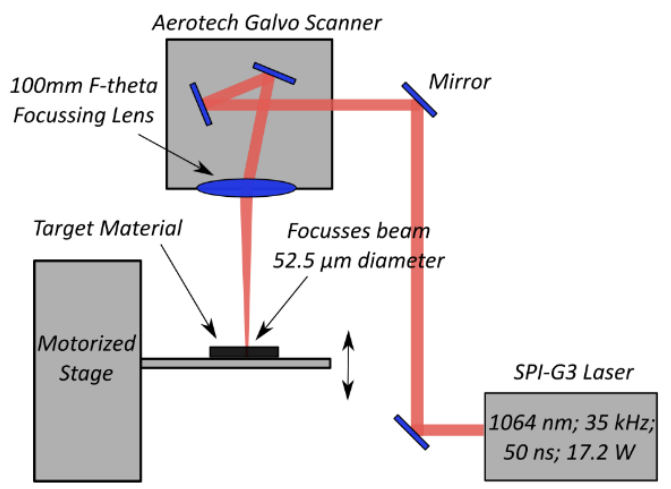

Fig. 4: Experimental Setup used for model calibration and validation.

The model presented above is calibrated and validated on two different metallic target materials that present material re-deposition for a wide range of process parameters. The first is a $\mathrm{Ni}$-based superalloy (Inconel 718 [34]), generally used in high temperature environments, such as gas turbine engines, due its high strength, high fatigue resistance and high resistance to oxidation. The second is a Ti alloy (Ti6Al4V, Al 6\% V4\% Ti bal.), a material with high strength to weight ratio and excellent oxidation resistance properties, often used for high performance application in the automotive, medical and aerospace industries. Both materials belong to a particular class of difficult-to-cut materials, which are usually processed industrially using non-conventional machining methods such as PLA.

In order to minimize the effect of surface roughness on the ablation process, samples are mechanically polished following standard metallographic sample preparation procedures with $\mathrm{SiC}$ papers to achieve a low surface roughness $(\mathrm{Ra}<50 \mathrm{~nm})$. Measurement of the sample surface is performed with a whitelight interferometer (Bruker Gt-i), with a resolution of $250 \mathrm{~nm}$, and analysed using an image analysis toolkit (MountainMap premium 7.2).

\subsection{Procedure for Model Calibration}

The calibration of the models presented above for single (Eq. 9) and overlapped trenches (Eq. 16) for a specific target material, is performed as an optimization problem over a set of experimentallyablated trenches, to minimize the difference between experimental $Z_{\text {exp }}(x, y, N)$ and modelled $Z_{\text {mod }}(x, y, N)$ surfaces. In practice, for a fixed material-laser (wavelength, power, repetition rate, beam diameter) combination, the model can be used over a wide a range of feed speeds (i.e. pulse separation distance, remembering that $\left.\delta x=V_{\text {feed }} / R\right)$ ).

For the prediction of single trenches (Eq. 9), two straight trenches at the lower and upper bounds of the feed speed range considered are required to calibrate the model parameters: the scaling coefficients $\left[\alpha^{+}, \alpha^{-}, \beta^{+}, \beta^{-}\right]$and the laser footprint $E(r)$. To extend the capabilities of the model for prediction of overlapped trenches (Eq. 16), the calibration of an additional coefficient $\left[k_{1}\right]$ is performed (Eq. 10), which requires an additional trench. 
The optimization problem for calibrating the model parameters can be formulated as a singleobjective optimization solvable with a non-linear least squares algorithm, with objective function set as

$$
F(\boldsymbol{x})=0.5 \sum\left(Z_{\text {exp }}\left(x_{i}, y_{j}, N\right)-Z_{\text {mod }}\left(x_{i}, y_{j}, N\right)^{2}\right)
$$

with input parameters $\boldsymbol{x}=\left[E(r), \alpha^{+}, \alpha^{-}, \beta^{+}, \beta^{-}, k_{1}\right.$ (optional)].

For the calibration, it must be ensured that the area considered for the calibration, $Z_{\text {exp }}\left(x_{i}, y_{j}, N\right)$, is one where the evolution of the surface has reached steady state (see Fig. 2 ) and the beam has been fully convoluted. This could represent a problem for very low feed speeds, as the short separation distance translates to a large number of pulses required to simulate the feature; furthermore, the high thermal input to the target material can result in the production of a high volume of melt/redeposition. As will be shown later, the performance of the model is limited in such scenarios, as the dynamics of melt movement (i.e. redeposition) can no longer be approximated as linear, and the influence of surface macro-geometry is significant. However, it must be noted that laser machining at such low feed speeds is often associated with excessive thermal damage [35], and higher feed speeds are generally preferred for real life applications.

The selection of an appropriate feed speed $\left(V_{\text {feed }}\right)$ range is therefore critical for the applicability of the model. Nevertheless, calibration of the framework to achieve control of the process via change of feed speed for fixed energy parameters (e.g. laser power) is still easier to implement compared to more traditional methods relying on changes of such energetic parameters.

In practice, both $\mathrm{Ni}$ - and $\mathrm{Ti}$ - superalloys samples have been calibrated and modelled in the feed speed range $300-1000 \mathrm{~mm} / \mathrm{s}$, intervals commonly used during laser machining. As will be shown later in the experimental results (section 5), in this range, the variation of depths achieved are significant (e.g. single trenches on Ni-superalloy at $70 \%$ max power results in $\sim 10 \mu \mathrm{m}$ depth at $300 \mathrm{~mm} / \mathrm{s}$ and $\sim 3 \mu \mathrm{m}$ depth at $1000 \mathrm{~mm} / \mathrm{s})$. The model can be calibrated in less than 20 minutes on a modern computer, and due to the simple experimental calibration required, it can be easily applied in industrial scenarios avoiding the need for time-consuming trial-and-error approaches where all processing conditions need to be considered.

The results shown in the next section present the calibrations and an overview of simple features modelled within the current framework, including single trenches at constant and variable feed speeds and overlapped trenches at different step-over distances. The tabulated error between experimental and modelled surfaces is reported as a \% difference in areas of redeposited and removed material taken from trench profiles (>2000 profiles, Fig. $5 \mathrm{~d}$ ). For overlapped trenches only a single error in areas is reported. The experimental error on the average profiles is defined as one standard deviation. Care should be taken when interpreting the errors, especially for overlapped trenches, as often, although the predicted amount of material removed/redeposited is close to the experimental value, the distribution of such material is not. For this reason, average profiles are also shown for selected cases.

\section{Results and Discussion}

This section presents the results and discussion for the calibration and the validation of the modelling framework presented above for single trenches (section 5.1) and overlapped trenches (section 5.2). 


\subsection{Validation of model for single trenches}

\subsubsection{Model calibration for single trenches}

Fig. 5 shows an example of calibration (Eq. 17) performed for a Ni-superalloy sample at $100 \%$ maximum power (17.2 W) for the modelling of single trenches (Eq. 9) using two single trenches at 300 $\mathrm{mm} / \mathrm{s}$ and $1000 \mathrm{~mm} / \mathrm{s}$ which represents the lower and upper bounds of the feed speed range considered.

For fixed parameter: Power, Wavelenght, Beam diameter, Repetition Rate, Material.

Calibrate model parameter $[E(r), a-, a+, \beta+, \beta-]$ for a given feed speed range by mathematical optimization (Eq. 19)

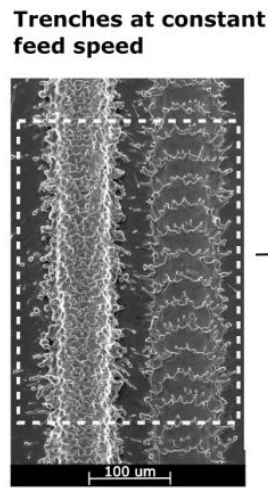

(a)

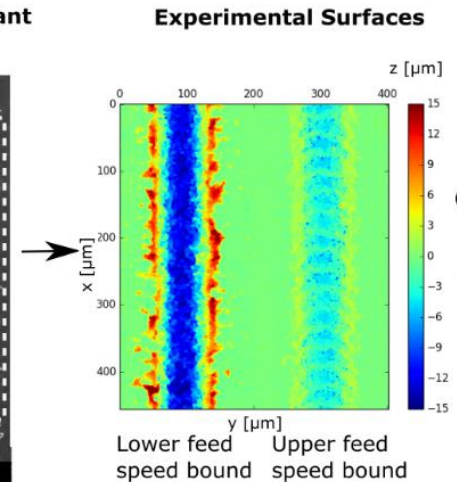

(b)

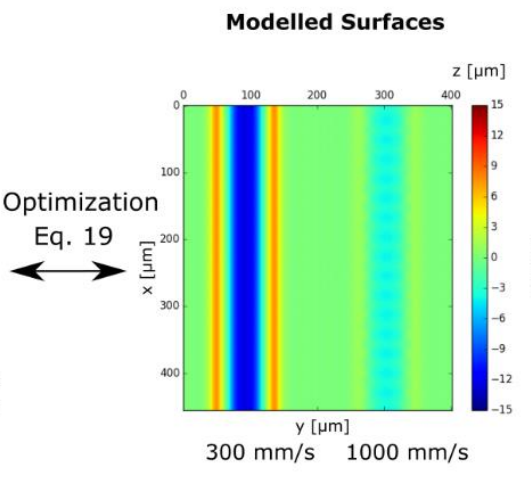

(c)

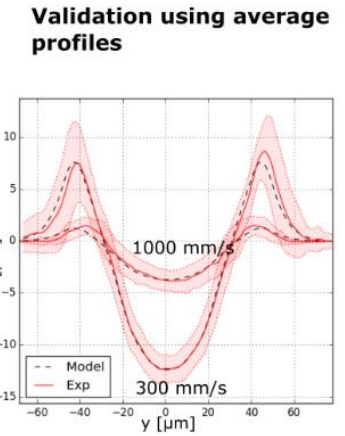

(d)

Fig. 5. Example of the steps needed for calibration of the single trench model using two single trenches at two extreme feed speed $V_{\text {feed }}(300 \mathrm{~mm} / \mathrm{s}$ and $1000 \mathrm{~mm} / \mathrm{s})$ : (a) SEM of experimental trenches, (b) Measurement of experimental surfaces, (c) Modelled surface after optimization, and (d) comparisons of modelled and experimental average section profiles. Note: workpiece material $\mathrm{Ni}$ - superalloy exposed to laser beam at $100 \%$ power (17.2W).

Excellent agreement is obtained between both features produced as can be observed from Fig. $5 \mathrm{~d}$, with an average error of $1.2 \%$ for material redeposition and $0.7 \%$ for material removal respectively (see Table 2). Overall, for the calibrations of all sample materials at the power levels investigated, Ni100\% (17.2 W), Ni70\% (12 W), Ni40\% (6.9 W), and Ti70\%(12 W), the average error is less than 4\% for material redeposition and $2.4 \%$ for material removal (see Table 2).

By evaluating the error reported in Table 2, there does not appear to be any significant skewness for the prediction of material redeposition and removal. It appears that, as one could have expected, that the error present during the model calibration for a particular feed speed range is carried over for the whole feed speed range considered (for example, looking at error in prediction of material removal for single trenches at $70 \%$ power); this emphasizes the need of performing a precise model calibration to achieve good surface predictions.

The good agreement between the profiles of the two trenches used for calibration (Fig. $5 \mathrm{~d}$ ) makes it possible to verify some of the assumptions and simplifications made during the formulation of the mathematical model. It is quite evident that the effect of feed speed, $V_{\text {feed }}$, can be approximated as a simple scaling in the $z$ direction, as the width of the trench is almost constant for the feed speed range considered.

This assumption is valid as long as the combination of material-power is kept constant. Comparing the laser footprint $E(r)$ obtained at different powers (Fig. 6) an increase of the width of the trenches can be observed as expected. This is the result of the Gaussian energy distribution of the laser pulses, as well as the possibility of exciting different mechanisms during the laser-material interaction (e.g. vaporization, phase explosion, fragmentation). 
Furthermore, when comparing the values of the calibrated model parameters (see Table 1), the values of the non-linear coefficients $\beta^{-}$and $\beta^{+}$indicate an increase of material removal/redeposition for subsequent pulses. This phenomenon is consistent with the current general understanding and modelling of incubation effects using logarithmic laws (which also predicts an increase of material removal [16]).

In the next subsections, results for prediction of single trenches at constant and variable feed speeds are presented for both $\mathrm{Ni}$-and $\mathrm{Ti}$ - superalloy target materials at different power levels.

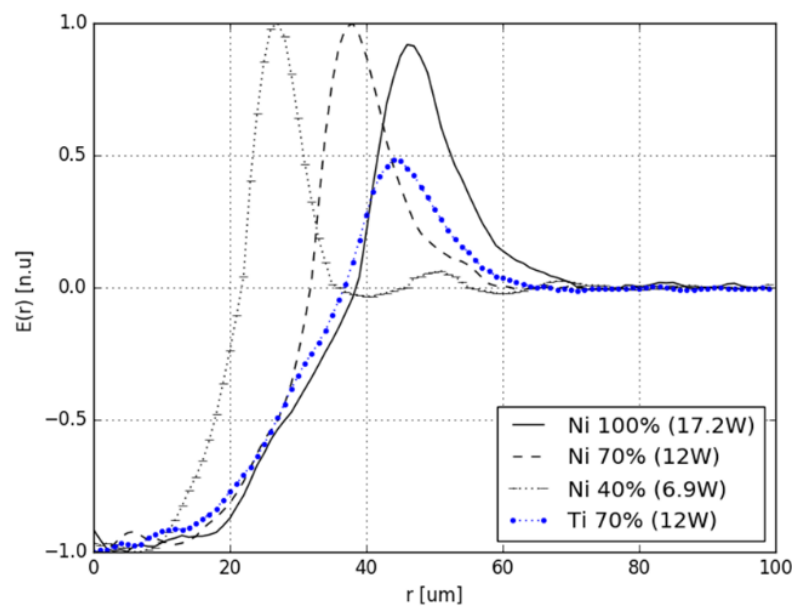

Fig. 6. Calibrated laser footprint $\mathrm{E}(r)$ for single trenches on $\mathrm{Ni}$ - and Ti- superalloy samples for fixed material-power combinations.

Table 1 Calibration coefficients for single trenches $\mathrm{Ni}$ - and Ti-superalloy samples for fixed material-power combinations.

\begin{tabular}{|c|c|c|c|c|c|}
\hline Material & $\begin{array}{c}\text { Power } \\
\%(\mathrm{~W})\end{array}$ & $\alpha^{-}$ & $\beta^{-}$ & $\alpha^{+}$ & $\beta^{+}$ \\
\hline \multirow{3}{*}{ Ni-alloy } & $\begin{array}{c}100 \\
(17.2)\end{array}$ & 4.78 & 0.110 & 25.7 & 0.534 \\
\cline { 2 - 6 } & $70(12)$ & 6.81 & 0.197 & 28.7 & 0.553 \\
\cline { 2 - 6 } & $40(6.9)$ & 9.12 & 0.242 & 35.6 & 0.605 \\
\hline Ti-alloy & $70(12)$ & 9.65 & 0.180 & 23.2 & 0.345 \\
\hline
\end{tabular}

\subsubsection{Simulation of single trenches at constant and variable feed speed.}

Single trenches at constant feed speed, $V_{\text {feed }}$, and different power levels have been modelled for workpiece samples made of $\mathrm{Ni}$ - and $\mathrm{Ti}$ - superalloys.

In Fig. 7 the maximum depth and redeposition height (averaged along the two peaks) is plotted against feed speed, together with the prediction of the model, verifying the consistent behaviour (i.e. power law relationship, eq. 8) of the ablation process within the feed speed range considered.

Generally, good agreement is achieved for all cases considered, with an average error of less than $8 \%$ (see Table 2). Trenches used for the optimization have the smallest error as expected, while the highest error is generally obtained for lower values of feed speed. 
By observing the surface prediction from the longitudinal profiles shown in Fig. 8a in the constant feed speed sections at $300 \mathrm{~mm} / \mathrm{s}$ and $800 \mathrm{~mm} / \mathrm{s}$ the model can be further evaluated.

High feed speeds generally result in better prediction. In particular, the model is able to correctly simulate the local roughness resulting from the overlapping between pulses, which is the result of both material removal $E^{-}(r)$ and redeposition $E^{+}(r)$. In contrast, the accuracy is less for the low feed speed case. This is due to the random nature of material ejection and subsequent approximation of the laser footprint as axisymmetric. This phenomenon is evident by looking at the experimental error bars for trenches produced at low feed speed, where a significant volume of melt is produced and ejected. Nevertheless, good average predictions are still obtained for this cases by looking at the average errors reported in Table 2.

A similar discussion can be made about the prediction of maximum redeposition height. It is evident from the surfaces shown in Fig. $8 \mathrm{~b}$ that the nature of redeposition (as pile-up of ejected material) appears quite random and results in a very uneven surface, which is difficult to simulate locally, so that only average predictions can, therefore, be made. 
Table 2: Error for single trenches at different feed speed and powers for Ni-and Ti-superalloy samples.

\begin{tabular}{|c|c|c|c|c|c|c|c|c|c|c|}
\hline \multirow[b]{2}{*}{ Material } & \multirow{2}{*}{$\begin{array}{l}\text { Power } \\
\%(W)\end{array}$} & \multicolumn{9}{|c|}{ Feed speed $(\mathrm{mm} / \mathrm{s})$} \\
\hline & & Error & $\begin{array}{c}300 \\
\text { (calib.) }\end{array}$ & 400 & 500 & 600 & 700 & 800 & 900 & $\begin{array}{c}1000 \\
\text { (calib.) }\end{array}$ \\
\hline \multirow{9}{*}{ Ni-alloy } & $\begin{array}{c}100 \\
(17.2)\end{array}$ & Avg. & $0.4 \%$ & $10.9 \%$ & $9.7 \%$ & $6.2 \%$ & $4.9 \%$ & $2.9 \%$ & $4.6 \%$ & $-0.8 \%$ \\
\hline & & Red. & $0.3 \%$ & $4.5 \%$ & $5.2 \%$ & $2.1 \%$ & $-2.7 \%$ & $-3.1 \%$ & $-4.0 \%$ & $-2.1 \%$ \\
\hline & & Rem. & $0.1 \%$ & $6.4 \%$ & $4.5 \%$ & $4.1 \%$ & $7.4 \%$ & $6.0 \%$ & $8.6 \%$ & $1.3 \%$ \\
\hline & $70(12)$ & Avg. & $-1.2 \%$ & $7.9 \%$ & $8.6 \%$ & $5.0 \%$ & $3.0 \%$ & $3.8 \%$ & $2.5 \%$ & $-1.3 \%$ \\
\hline & & Red. & $0.2 \%$ & $11.4 \%$ & $11.7 \%$ & $8.6 \%$ & $6.9 \%$ & $7.7 \%$ & $4.1 \%$ & $4.5 \%$ \\
\hline & & Rem. & $-1.4 \%$ & $-3.5 \%$ & $-3.5 \%$ & $-3.6 \%$ & $-3.9 \%$ & $-3.9 \%$ & $-1.6 \%$ & $-5.8 \%$ \\
\hline & $40(6.9)$ & Avg. & $0.8 \%$ & $5.7 \%$ & $-5.7 \%$ & $-5.2 \%$ & $1.5 \%$ & $-5.9 \%$ & $-8.8 \%$ & $-16.1 \%$ \\
\hline & & Red. & $-1.9 \%$ & $-2.9 \%$ & $-10.1 \%$ & $-7.4 \%$ & $-4.5 \%$ & $-9.1 \%$ & $-16.5 \%$ & $-21.5 \%$ \\
\hline & & Rem. & $2.7 \%$ & $8.6 \%$ & $4.4 \%$ & $2.2 \%$ & $6.0 \%$ & $3.2 \%$ & $7.7 \%$ & $5.4 \%$ \\
\hline \multirow{3}{*}{ Ti-alloy } & $70(12)$ & Avg. & $-0.1 \%$ & $7.4 \%$ & $3.8 \%$ & $2.4 \%$ & $2.3 \%$ & $-3.7 \%$ & $-2.6 \%$ & $0.9 \%$ \\
\hline & & Red. & $0.0 \%$ & $-1.2 \%$ & $-2.7 \%$ & $-4.4 \%$ & $-2.5 \%$ & $-6.3 \%$ & $-5.3 \%$ & $-1.7 \%$ \\
\hline & & Rem. & $-0.1 \%$ & $8.6 \%$ & $6.5 \%$ & $6.8 \%$ & $4.8 \%$ & $2.6 \%$ & $2.7 \%$ & $2.6 \%$ \\
\hline
\end{tabular}
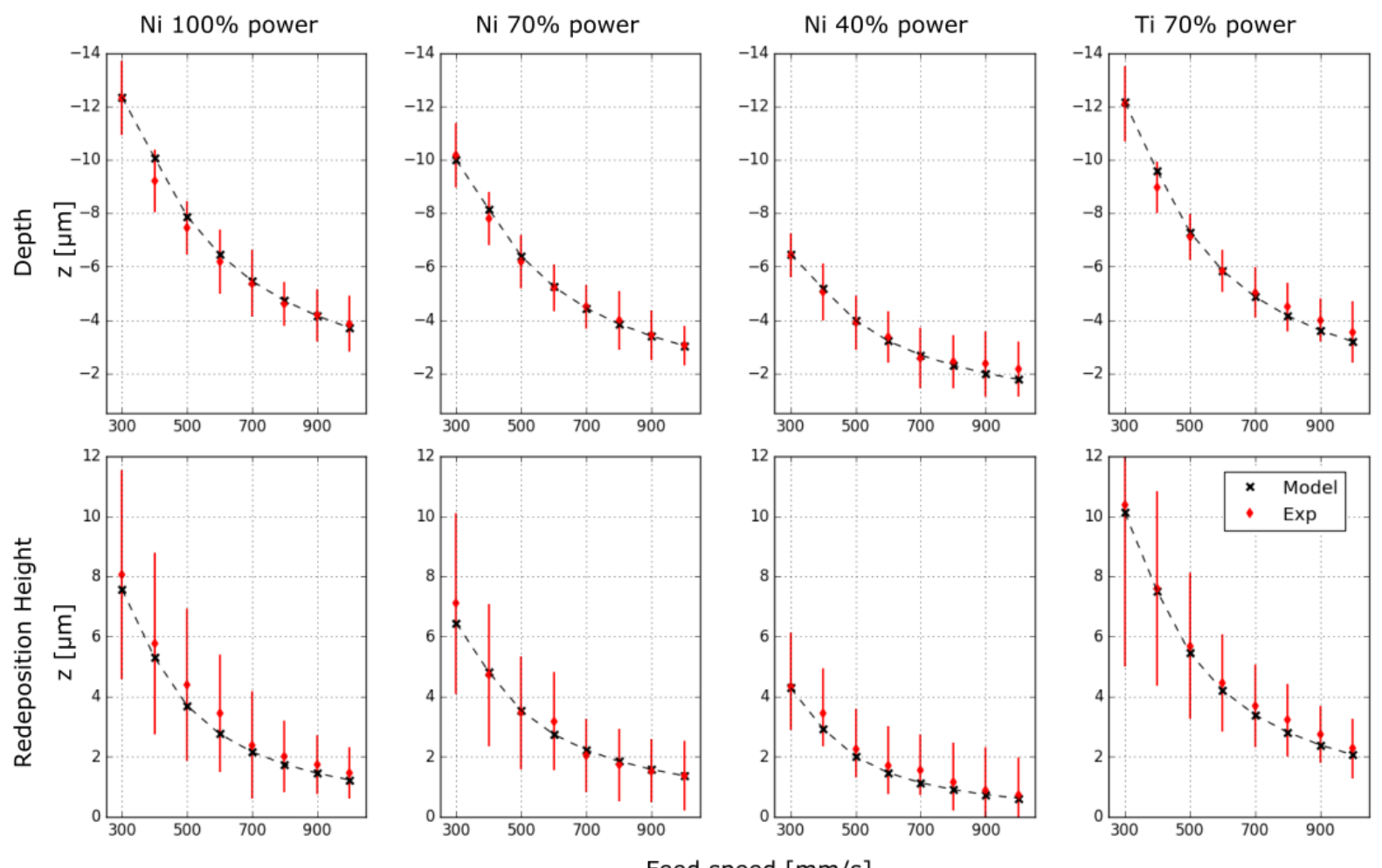

Feed speed $[\mathrm{mm} / \mathrm{s}]$

Fig. 7. Modelled and experimental values of maximum depth (a) and redeposition height (b) of single trenches for different feed speeds and powers for both $\mathrm{Ni}$ - and Ti-superalloys target workpieces

Fig. 8 also presents the results obtained for trenches produced at continuously varied (decreased) feed speed. The model is tested with the highest possible acceleration achievable by the system used (300 $000 \mathrm{~mm} / \mathrm{s}^{\wedge} 2$ ). Higher acceleration translates to higher approximation error within our modelling framework (eq. 6), but allows the laser to reach the desired target speed faster, limiting the error to a shorter path length, partially counterbalancing the negative effects. 
Results shown in Fig. 8 highlight that a generally good level of prediction can be obtained for both maximum depth and redeposition height, with the biggest error limited to the velocity ramp down, in particular at lower feed speeds.

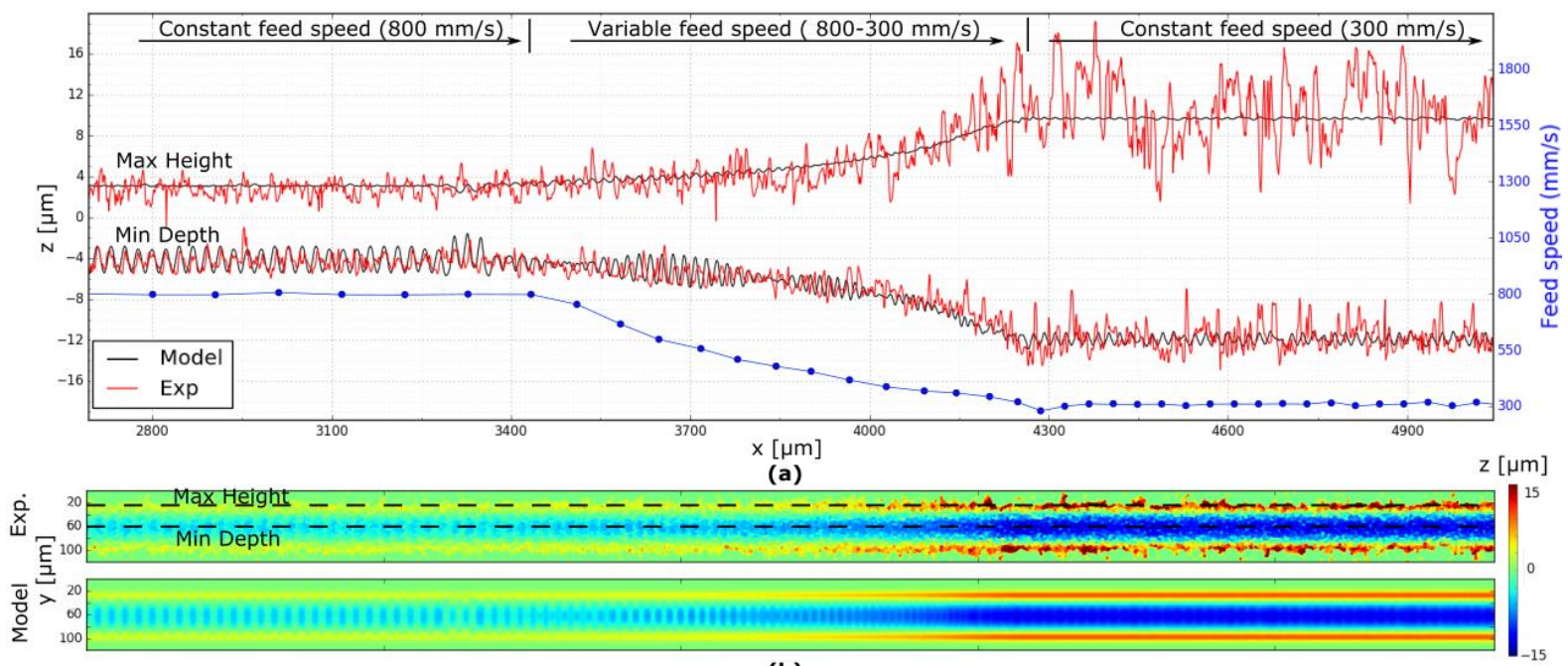

(b)

Fig. 8. Examples of single trenches at variable feed speed (300 to $800 \mathrm{~mm} / \mathrm{s}$ ) - Experimental vs. Modelled results: (a) Line profiles of maximum depth and redeposition height, and (b) top view of trenches. Ti- superalloy sample at $70 \%$ power.

The results presented in this section allow us to draw some initial conclusions with regards to the good performance of the model for the prediction of single trenches, laying the groundwork for the subsequent model modification aimed at the prediction of more complex features, such as overlapped trenches.

\subsection{Validation of model for overlapped trenches}

\subsubsection{Model calibration for overlapped trenches}

Prediction of overlapped trenches requires calibration of a modified model framework (Eq. 16). This is necessary as it has been observed that the original model underpredicted the removal rate (i.e. $\left.E^{-}(r)\right)$ of material previously redeposited. This is accounted for by introducing an additional linear scaling coefficient $k_{1}$ (Eq. 10). 
For fixed parameter: Power, Wavelenght, Beam diameter, Repetition Rate, Material. Optimize model parameter $\left[E(r), a_{-}, a+, \beta+, \beta-, k 1\right]$ for a given feed speed range.

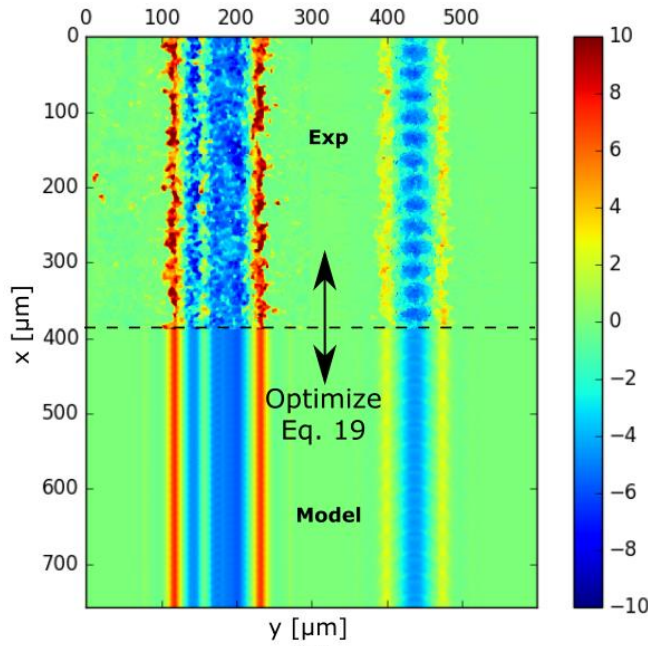

(a)
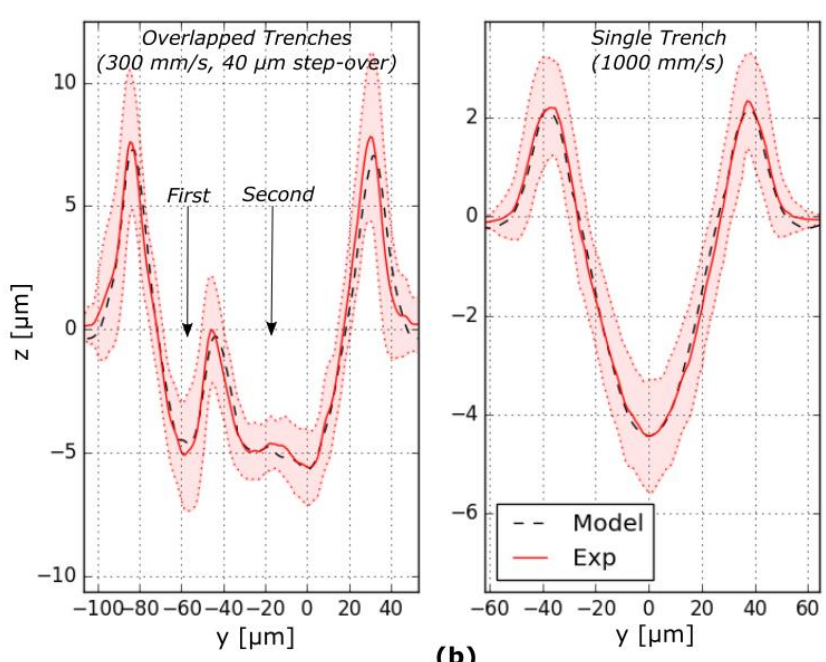

(b)

Fig. 9. Example of calibration for overlapped trenches modelling using 2 trenches at $300 \mathrm{~mm} / \mathrm{s}$ and 1 at $1000 \mathrm{~mm} / \mathrm{s}$ : (a) experimental and modelled surfaces and (b) comparisons of average section profiles; Ti-superalloy sample at $70 \%$ power $(12 W)$.

Fig. 9 shows the profiles used for the calibration for the Ti-superalloy sample at $12 \mathrm{~W}$ (70\% power), by using a single trench at $1000 \mathrm{~mm} / \mathrm{s}$ and two overlapped trenches at $300 \mathrm{~mm} / \mathrm{s}$ with a step-over distance of $40 \mu \mathrm{m}$.

As can be observed, the implementation of the model does not influence the prediction of single trenches (i.e. the first of the overlapped trenches at $300 \mathrm{~mm} / \mathrm{s}$ and the single trench at $1000 \mathrm{~mm} / \mathrm{s}$ ). Furthermore, it appears to be able to predict the over-ablation of redeposited material correctly.

The value of the coefficients $k_{1}$ for $\mathrm{Ni}$ and Ti alloys target workpieces at $12 \mathrm{~W}$ (70\% power) are 2.54 and 4.13 respectively (see Table 3 ). The values of the coefficient $k_{1}$ are larger than 1 , implying an increase of removal rate for both target materials. Likely this is linked with the volume of material redeposited and the oxidation which takes place during PLA in atmospheric condition [31]. This result in a dramatic increase of absorptance due to the lower reflectance of the oxide compare to virgin metal, and therefore an increase of material removal rate [32][33]. Mechanical integrity of the redeposited layer is also likely to play a role in the apparent increase of ablation rate [19].

The larger value of the coefficient $k_{1}$ for the Ti-superalloy can also be explained on this premises by considering the higher volume of oxidized material, compared to Ni-superalloy, generally associated with laser machining of this material [31].

Table 3: Calibration coefficients for single trenches Ni- and Ti-superalloy samples for fixed material-power combinations.

\begin{tabular}{|c|c|c|c|c|c|c|}
\hline Material & $\begin{array}{c}\text { Power } \\
\%(\mathrm{~W})\end{array}$ & $\alpha^{-}$ & $\beta^{-}$ & $\alpha^{+}$ & $\beta^{+}$ & $k_{1}$ \\
\hline Ni-alloy & $70(12)$ & 2.80 & 0.105 & 6.13 & 0.417 & 2.54 \\
\hline
\end{tabular}




\begin{tabular}{|l|l|l|l|l|l|l|}
\hline Ti-alloy & $70(12)$ & 5.64 & 0.046 & 3.24 & 0.133 & 4.13 \\
\hline
\end{tabular}

\subsubsection{Simulation of overlapped trenches}

Fig. 10 show a selection of trenches for the $\mathrm{Ni}$ - (at $300 \mathrm{~mm} / \mathrm{s}$ and $600 \mathrm{~mm} / \mathrm{s}$ ) and Ti- superalloy (at 300 $\mathrm{mm} / \mathrm{s}$ ) samples at $70 \%$ power and different step-over distances,

It is evident that prediction of overlapped trenches with large step-over distances $(30 / 35 \mu \mathrm{m})$ produces results in good agreement with the experimental data (<6\% error, see Table 4); on the other hand, the model is not able to perfectly capture the asymmetry of the process in the case of overlapped trenches with small step-over distances $(15 / 20 \mu \mathrm{m})$, where the distribution of material is not predicted correctly.

Similar errors are reported for the particular case of totally overlapped trenches (i.e. Oum step-over); although the features are correctly modelled as symmetric, and low errors ( $<7 \%$ error, see Table 4$)$ are obtained for these cases, which signifies a correctly modelled total volume of material removed and redeposited, the distribution of material is predicted with less accuracy.

The origin of these errors can be traced back to surface conditions (i.e. inclination angle) which influence the dynamic of melt movement. In the case of small step-over distance, the surface induces asymmetry in the distribution of forces (recoil pressure, thermal gradients) which lead to the ejection of material with a-non-axisymmetric distribution. In the case of Oum step-over, the model fails to account for the higher aspect ratio of the trenches compared to an originally flat surface.

Whilst, on the one hand, this could limit the applicability of the model for machining deep structures, (which lies outside the scope of this framework), on the other, it is quite interesting that the process and dynamics of melt movement can be described by a simple linear model for different overlaps, and the prediction of "shallow" features is achievable with good success. 

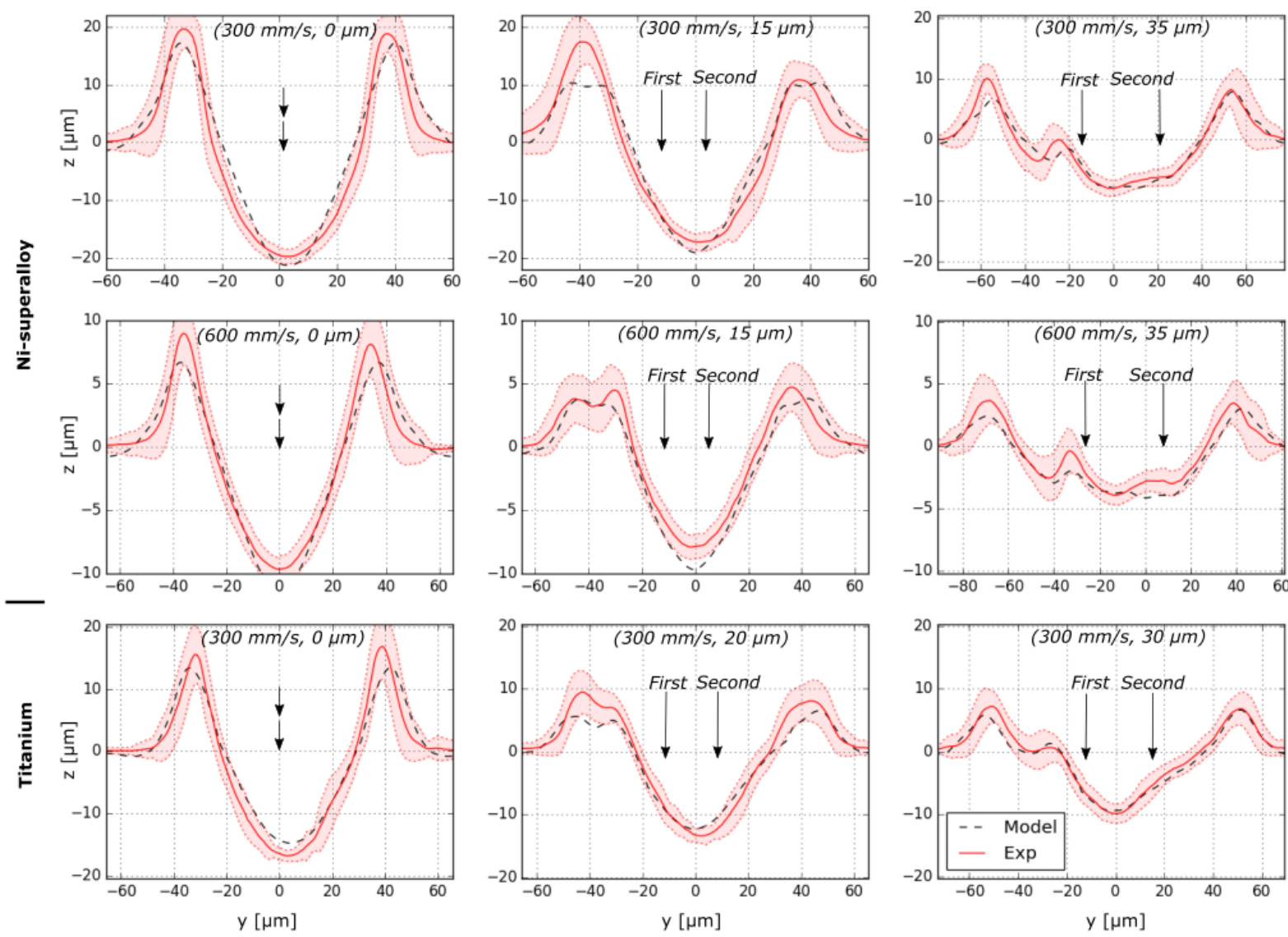

Fig. 10. Comparisons of average section profiles of overlapped trenches at different step-over distances: (top) Ni-and (bottom) Ti-superalloy samples at $70 \%$ power.

Table 4: Error for overlapped trenches at different step-over distances for $\mathrm{Ni}$ - and Ti-superalloy samples at 70\% power.

\begin{tabular}{|c|c|c|c|c|c|c|c|}
\hline \multirow{2}{*}{ Material } & \multirow{2}{*}{$\begin{array}{l}\text { Feed Speed } \\
(\mathrm{mm} / \mathrm{s})\end{array}$} & \multicolumn{4}{|c|}{ Step-over distance (um) } & \multirow[b]{2}{*}{$35 \mu \mathrm{m}$} & \multirow[b]{2}{*}{$45 \mu \mathrm{m}$} \\
\hline & & $0 \mu \mathrm{m}$ & $5 \mu \mathrm{m}$ & $15 \mu \mathrm{m}$ & $25 \mu \mathrm{m}$ & & \\
\hline \multirow{2}{*}{$\begin{array}{l}\text { Ni-based } \\
\text { alloy }\end{array}$} & 300 & $3.8 \%$ & $4.1 \%$ & $6.5 \%$ & $0.9 \%$ & $2.9 \%$ & $\begin{array}{l}0.4 \% \\
\text { (calib.) }\end{array}$ \\
\hline & 600 & $5.1 \%$ & $8.2 \%$ & $7.9 \%$ & $8.1 \%$ & $10.3 \%$ & $15.5 \%$ \\
\hline \multirow{2}{*}{$\begin{array}{l}\text { Ti-based } \\
\text { alloy }\end{array}$} & \multirow[b]{2}{*}{300} & $0 \mu \mathrm{m}$ & $10 \mu \mathrm{m}$ & $20 \mu \mathrm{m}$ & $30 \mu \mathrm{m}$ & $40 \mu \mathrm{m}$ & 1 \\
\hline & & $6.7 \%$ & $8.5 \%$ & $17.4 \%$ & $4.1 \%$ & $\begin{array}{l}0.6 \% \\
\text { (calib.) }\end{array}$ & \\
\hline
\end{tabular}

\section{Conclusion}

Modelling of Pulsed Laser Ablation (PLA) of metallic materials for the prediction of surface geometry after machining is a challenging task. The presence of significant volumes of melt ejected and redeposited, in particular for nanosecond PLA of metallic materials, as well as the non-linear 
interaction between successive pulses, often makes mathematical approximation necessary to allow an experimentally accurate prediction material removal.

In this paper, a novel modelling framework has been presented, allowing, from a simple experimental calibration, accurate prediction of features produced by a scanned laser beam in materials which experience redeposition, validated on two metallic materials ( $\mathrm{Ni}$ - and Ti- superalloy).

The main contributions of this paper are:

- The non-linear effect of the interaction between successive pulses from a scanned laser beam can be assumed to reach a steady state after a finite number of pulses. Effectively, this allows us to represent the process dynamics as steady state and to formulate a simplified mathematical formulation that models the change in surface depth \height as the convolution of a radial function $E(r)$ representing the scanned laser beam. Furthermore, the behaviour of molten/redeposited material during PLA of metals can also be encapsulated in this approach. This allows to account for and predict the resulting effect of the molten material on the final surface, and is the main novelty of the framework.

- When the laser beam is scanned along straight path to produce single trenches, the effect of feed speed (i.e. intra-pulse separation distances), result in a simple scaling of the footprint function $E(r)$, and the non-linear interaction between successive pulses can be expressed as function of the feed speed through a power law relationship for a fixed set of laser-material (wavelength, power, repetition rate, beam diameter) combination. Prediction of straight trenches at constant and variable feed speed has been successfully reported with an average error of less than $8 \%$.

- For overlapped trenches, it has been found that redeposited material is removed at a higher rate compared to virgin material, likely due to changes in microstructure and chemical composition. This phenomenon can be incorporated into the model using a simple linear scaling of the footprint function $\mathrm{E}(\mathrm{r})$. Good predictions are obtained for overlapped trenches at large step-over distances, with errors of less than $10 \%$. This further demonstrates that the dynamics of melt movement can be predicted by a simple linear model, and the influence of the surface condition (i.e. inclination angle) is limited. In contrast, overlapped trenches at low step-over distance lead to problems in predicting the correct distribution of material but maintain a similar level of errors (less than $10 \%$ ). As expected, the surface introduces nonlinearity/asymmetry in the dynamics of the forces responsible for the movement of molten material, rendering the process more difficult to simulate.

The results shown and the framework presented in this paper could provide a significant step forward in process simulation of PLA for prediction of surfaces during machining of shallow features in materials where redeposition occurs. 


\section{Reference}

[1] Knowles, M. R H, G. Rutterford, D. Karnakis, and A. Ferguson, 2007. "Micro-Machining of Metals, Ceramics and Polymers Using Nanosecond Lasers." International Journal of Advanced Manufacturing Technology, 33 (1-2): 95-102. doi:10.1007/s00170-007-09672.

[2] Butler-Smith, P. W., Axinte, D. A., Pacella, M., and Fay, M. W, 2013. "Micro/Nanometric Investigations of the Effects of Laser Ablation in the Generation of Micro-Tools from Solid CVD Diamond Structures." Journal of Materials Processing Technology 213 (2): 194-200. doi:10.1016/j.jmatprotec.2012.08.010.

[3] Feng, Q., Picard, Y. N., McDonald, J. P. , Van Rompay, P. A., Yalisove, S. M., and Pollock, T. M., 2006. "Femtosecond Laser Machining of Single-Crystal Superalloys through Thermal Barrier Coatings." Materials Science and Engineering A 430 (1-2): 203-207. doi:10.1016/j.msea.2006.05.104.

[4] Stafe, M., Aurelian M., and Niculae, N. P., 2014, "Pulsed Laser Ablation of Solids: Basics, Theory and Applications", Springer Series in Materials Science Vol. 53, Springer Springer Science \& Business Media, Chap. 2. ISBN: 9783642409783

[5] Sundaram, S. K., and Mazur, E., 2002. "Inducing and Probing Non-Thermal Transitions in Semiconductors Using Femtosecond Laser Pulses." Nature Materials 1: 217-224. doi:10.1038/nmat767.

[6] Bogaerts, A., and Chen, Z., 2005. "Effect of Laser Parameters on Laser Ablation and LaserInduced Plasma Formation: A Numerical Modeling Investigation." Spectrochimica Acta Part B Atomic Spectroscopy 60 (9-10): 1280-1307. doi:10.1016/j.sab.2005.06.009.

[7] Demos, S. G., Negres, R. A., Raman, R. N., Feit, M. D., Manes, K. R., and Rubenchik, A M., 2015. "Relaxation Dynamics of Nanosecond Laser Superheated Material in Dielectrics." Optica 2 (8): 765-772. doi:10.1364/OPTICA.2.000765.

[8] Stafe, M, 2012. "Theoretical Photo-Thermo-Hydrodynamic Approach to the Laser Ablation of Metals." Journal of Applied Physics 112 (12): 123112 1-7. doi:10.1063/1.4771692.

[9] Cadot, G. B. J., Billingham, J., and Axinte, D. A., 2017. "A Study of Surface Swelling Caused by Graphitisation during Pulsed Laser Ablation of Carbon Allotrope with High Content of Sp3 Bounds." Journal of Physics D: Applied Physics 50 (24). doi:10.1088/13616463/aa70fe.

[10] Schwarz-Selinger, T., Cahill, D. G., Chen, S. C., Moon, S. J., and Grigoropoulos ,C. P., 2001. "Micron-Scale Modifications of Si Surface Morphology by Pulsed-Laser Texturing." Physical Review B - Condensed Matter and Materials Physics 64 (15): 155323 1-7. doi:10.1103/PhysRevB.64.155323. 
[11] Tani, G., Orazi, L., Fortunato, A., and Cuccolini, G., 2008. "Laser Ablation of Metals: A 3D Process Simulation for Industrial Applications." Journal of Manufacturing Science and Engineering 130: 031111 1-11. doi:10.1115/1.2917326.

[12] Samant, A. N., and Dahotre, N. B., 2010. "Three-Dimensional Laser Machining of Structural Ceramics." Journal of Manufacturing Processes 12 (1): 1-7. doi:10.1016/j.jmapro.2010.01.001.

[13] St-Onge, L., 2002. "A Mathematical Framework for Modeling the Compositional Depth Profiles Obtained by Pulsed Laser Ablation." J. Anal. At. Spectrom. 17 (9): 1083-89. doi:10.1039/B203024C.

[14]Parandoush, P., and Altab, H., 2014. "A Review of Modeling and Simulation of Laser Beam Machining." International Journal of Machine Tools and Manufacture 85: 135-45. doi:10.1016/j.ijmachtools.2014.05.008.

[15] Vora, H. D., and Narendra, B. D., 2015. "Surface Topography in Three-Dimensional Laser Machining of Structural Alumina." Journal of Manufacturing Processes 19: 49-58. doi:10.1016/j.jmapro.2015.04.002.

[16] Jee, Y., Becker, M. F., and Walser, R. M.,1988. "Laser-Induced Damage on Single-Crystal Metal Surfaces." Journal of the Optical Society of America B 5 (3): 648-659. doi:10.1364/JOSAB.5.000648.

[17] Mannion, P. T., Magee, J., Coyne, E., O'Connor, G. M., and Glynn, T. J., 2004. "The Effect of Damage Accumulation Behaviour on Ablation Thresholds and Damage Morphology in Ultrafast Laser Micro-Machining of Common Metals in Air." Applied Surface Science 233 (1-4): 275-87. doi:10.1016/j.apsusc.2004.03.229.

[18 ] Benocci, R., Batani, D., and Roman, H. E., 2019. "Incubation Models for UnderThreshold Laser Ablation with Thermal Dissipation." Applied Physics B: Lasers and Optics 125 (2). doi:10.1007/s00340-019-7132-0.

[19] Wu, C., Christensen, M. S., Savolainen, J. M., Balling, P., and Zhigilei, L. V., 2015. "Generation of Subsurface Voids and a Nanocrystalline Surface Layer in Femtosecond Laser Irradiation of a Single-Crystal Ag Target." Physical Review B - Condensed Matter and Materials Physics 91 (3): 1-14. doi:10.1103/PhysRevB.91.035413.

[20] Galasso, G., Kaltenbacher, M., Tomaselli, A., and Scarpa, D., 2015. “A Unified Model to Determine the Energy Partitioning between Target and Plasma in Nanosecond Laser Ablation of Silicon." Journal of Applied Physics 117 (12): 123101 1-11 doi:10.1063/1.4915118.

[21] Ancona, A., Röser, F., Rademaker, K., Limpert, J., Nolte, S., and Tünnermann, A., 2008. "High Speed Laser Drilling of Metals Using a High Repetition Rate, High Average Power Ultrafast Fiber CPA System." Optics Express 16 (12): 8958- 8568. doi:10.1364/OE.16.008958. 
[22] Holmes, A. S., Onischenko, A. I., George, D. S., and Pedder, J. E., 2005. "Modelling of SolidState and Excimer Laser Processes for 3D Micromachining." SPIE Proceeding 5713: 1-10. doi:10.1117/12.600632.

[23] Xiao, S., Gurevich, E. L., and Ostendorf, A., 2012. "Incubation Effect and Its Influence on Laser Patterning of ITO Thin Film." Applied Physics A: Materials Science and Processing 107 (2): 333-338. doi:10.1007/s00339-012-6820-y.

[24] Axinte, D. A., Billingham, J., and Guillerna, A. B., 2017. "New Models for Energy Beam Machining Enable Accurate Generation of Free Forms." Science Advances 3: 1-8. doi:10.1126/sciadv.1701201.

[25] Cadot, G. B. J., Billingham, J., and Axinte, D. A., 2016. "Continuous Trench; Pulsed Laser Ablation for Micro-Machining Applications." International Journal of Machine Tools and Manufacture 107: 8-20. doi:10.1016/j.ijmachtools.2016.04.011.

[26] Chen, S. C., Cahill, D. G., Grigoropoulos, and C. P., 2000. "Melting and Surface Deformation in Pulsed Laser Surface Micromodification of Ni-P Disks." Journal of Heat Transfer 122 (1): 107-112. doi:10.1115/1.521441.

[27] Kirichenko, N A., 2009. "Large-Scale Structures Produced on Metal Surfaces by Multiple Laser Pulses." Quantum Electronics $39 \quad$ (5): 442-48. doi:10.1070/Qe2009v039n05abeh013921.

[28] Gilbert, D., Stoesslein, M., Axinte, D., Butler-Smith, P., and Kell, J., 2014. “A Time Based Method for Predicting the Workpiece Surface Micro-Topography under Pulsed Laser Ablation." Journal of Materials Processing Technology 214 (12): 3077-3088. doi:10.1016/j.jmatprotec.2014.07.008.

[29] Wang, Y., Ninggang, S., Getachew, K. B., and Crystal, L. P., 2017. “Modeling Pulsed Laser Ablation of Aluminum with Finite Element Analysis Considering Material Moving Front." International Journal of Heat and Mass Transfer 113: 1246-53. doi:10.1016/j.ijheatmasstransfer.2017.06.056.

[30] Lutey, A. H. A., 2013. "An Improved Model for Nanosecond Pulsed Laser Ablation of Metals." Journal of Applied Physics 114 (8). doi:10.1063/1.4818513.

[31] Bandyopadhyay, S., Sarin Sundar, J. K., Sundararajan, G., and Joshi, S. V., 2002. "Geometrical Features and Metallurgical Characteristics of Nd:YAG Laser Drilled Holes in Thick IN718 and Ti-6Al-4V Sheets." Journal of Materials Processing Technology 127 (1): 83-95. doi:10.1016/S0924-0136(02)00270-4.

[32] Antończak, A. J., Łukasz, S., Trzcinski,A., Kinzhybalo, V. V., Łazarek, L. K., and Krzysztof M. A., 2015. "Laser-Induced Oxidation of Titanium Substrate: Analysis of the Physicochemical Structure of the Surface and Sub-Surface Layers." Applied Surface Science 325 (C): 217-26. doi:10.1016/j.apsusc.2014.11.062. 
[33] Vorobyev, A. Y., and Chunlei, G., 2009. "Femtosecond Laser Blackening of Metals." Midwest Symposium on Circuits and Systems. IEEE: 905-8.

doi:10.1109/MWSCAS.2009.5235913.

[34] 2018 SpecialMetals High Performance Alloys Literature

(http://www.specialmetals.com/assets/smc/documents/inconel_alloy_718.pdf)

[35] Bauer, F., Michalowski, A., Kiedrowski, T., and Nolte, S., 2015. "Heat Accumulation in Ultra-Short Pulsed Scanning Laser Ablation of Metals." Optics Express 23 (2): 1035-1043. doi:10.1364/OE.23.001035. 